\title{
Testicule non descendu : le point sur les causes et les traitements
}

\author{
R. MIEUSSET
}

Centre de Stérilité Masculine, Groupe de Recherche en Fertilité Humaine, Service d'Urologie-Andrologie, Hôpital La Grave, Toulouse

\section{INTRODUCTION}

La non-descente du testicule est une malformation congénitale dont la ou les causes restent toujours inconnues. Ceci n'est pas vraiment surprenant dans la mesure où on ignore toujours quels sont le ou les mécanismes impliqués dans cette migration et quels en sont les déclencheurs, les intermédiaires, les régulateurs, les organes cibles et les agents. Cependant, les travaux sont nombreux, et si ceux rapportés ces trois dernières années, n'apportent aucune réponse positive quant aux causes de cette non-descente, ils donnent des réponses clairement négatives chez l'homme et, ils enrichissent de façon très importante les différents modèles conceptuels ou animaux sur lesquels reposent notre compréhension actuelle de la migration testiculaire. Ces différents travaux seront revus dans le chapitre suivant intitulé "Causes de la non-descente du testicule".

Les deux conséquences les plus graves de la non-descente testiculaire sont représentées par le risque de cancer du testicule et d'infécondité. Pour prévenir au maximum ces deux risques, le "traitement " proposé pour cette absence de migration testiculaire est d'amener le ou les testicules en situation scrotale basse permanente (localisation physiologique) soit par l'apport exogène de certaines hormones, soit par chirurgie. Si la principale raison de ce traitement a été de permettre le dépistage précoce d'une tumeur, les très bons résultats, en terme de survie des hommes atteints de cancers du testicule, consécutifs aux médications cytotoxiques ont, peu à peu, amené la question de la fertilité associée à ce traitement de la non-descente testiculaire. Ce questionnement porte autant sur les résultats immédiats de chaque type de traitement, comme la position finale du testicule, que sur les résultats à long terme, tels que spermatogenèse et fertilité, mais aussi sur les effets secondaires. Les données récentes dans ce domaine sont rapportées dans le chapitre intitulé "Traitements de la non-descente du testicule".

\section{CAUSES DE LA NON DESCENTE TESTICULAIRE.}

Bien que l'on pense que la cause la plus fréquente de la non-descente testiculaire soit un défaut de la sécrétion prénatale d'androgènes, d'autres étiologies sont envisagées, comme les causes génétiques, et des facteurs de risque sont recherchés.

\section{Causes génétiques.}

Plusieurs gènes ou familles de gènes semblent être des candidats potentiels à une " multicausalité" de la non-descente testiculaire.

Correspondance : Roger Mieusset, Centre de Stérilité Masculine, Groupe de Recherche en Fertilité Humaine, Service d'Urologie-Andrologie, Hôpital La Grave, 31052 Toulouse Cedex, France.

e-mail:mieusset@cict.fr 


\section{a) Les gènes Homeobox.}

Les gènes Homeobox (Hox) ont un rôle clé dans la morphogenèse des structures segmentaires le long de l'axe primaire antéro-postérieur de l'embryon. Ces gènes, qui sont exprimés dans de nombreuses cellules dont celles du système urogénital, agissent comme des régulateurs majeurs qui spécifient l'identité axiale et contrôlent la croissance et la différentiation des cellules reliées par leur position. Ces gènes présentent une remarquable stabilité évolutive ; ainsi, les gènes murins Hox $a, b$, c et d (respectivement portés par les chromosomes 6,11 , 5 et 2) correspondent aux gènes humains HOX A, B, C et D (respectivement portés par les chromosomes 7, 17, 12 et 2). De façon plus spécifique, Hoxa10 est exprimé dans les champs postérieurs chez l'embryon de souris en développement, incluant, entre autres, le mésoderme intermédiaire qui donnera naissance à une partie de l'appareil uro-génital.

La délétion ciblée du gène Hoxa10 [59, 57] permet, entre autres, l'obtention de souris mâles homozygotes viables et déficientes pour les transcripts du gène Hoxa10 (Hoxa10-déficientes). Toutes les souris mâles Hoxa10-déficientes présentent un testicule non descendu (TND) soit bilatéral, et dans tous les cas à localisation abdominale [59], soit unilatéral et toujours à gauche, ou bilatéral et associé à une absence plus ou moins complète du sac scrotal et à une réduction de la distance ano-génitale [57]. L'analyse histologique des testicules montre la dynamique des lésions cellulaires classiquement décrite en cas de " cryptorchidie " pour arriver à l'âge adulte à une réduction du diamètre des tubules séminifères et à la présence de tubes séminifères dépourvus de cellules germinales. Bien qu'à l'âge adulte la virilisation de ces mâles soit normale, $40 \%$ sont stériles et ce pourcentage croît avec l'âge. Il a aussi été noté dans une autre étude [5] que le même type de délétion était associé à une transformation partielle du canal déférent proximal en épididyme distal, de l'épididyme distal en proximal, ainsi qu'à des modifications de la prostate.

Cette délétion du gène Hoxa10 est associée en post-natal chez ces mâles homozygotes à une absence de raccourcissement du gubernaculum testis et de prolifération de son extrémité bulbaire $[59,57]$, deux phénomènes qui seraient responsables de la phase de migration transinguinale du testicule qui se produit dans les deux à trois semaines suivant la naissance chez la souris. Il semble donc que l'absence complète du gène Hoxa 10 chez la souris mâle, entraîne après la naissance un mauvais développement du gubernaculum testis et par conséquent une non-descente testiculaire [59].

Cependant, le nerf génito-fémoral (GFN) qui innerve le gubernaculum testis et le muscle crémaster, serait le médiateur de l'action des androgènes dans la descente testiculaire chez la souris, le gubernaculum testis étant dépourvu de récepteurs aux androgènes [34]. Pour jouer ce rôle, le gubernaculum testis doit être innervé par un GFN fonctionnel qui naît des premier et deuxième segments lombaires de la moelle épinière [34]. Or, chez les mâles homozygotes Hoxa10-déficients, il existe aussi, entre autres, une morphogenèse anormale à type de transformation homéotique antérieure (i.e. thoracique) du segment lombaire de la moelle épinière [57]. Les auteurs ont donc suggéré que la non-descente testiculaire serait en fait la conséquence d'une spécification incorrecte des motoneurones du GFN et/ou d'une innervation anormale du tissu cible [57].

Une étude préliminaire des mutations du gène Homeobox HOXA10 a été récemment rapportée chez l'homme [39] : les résultats de l'analyse d'échantillons de gubernaculum testis, sac herniaire et muscle crémaster prélevés au décours de l'orchidopexie chez des enfants ayant un TND uni ou bilatéral furent comparés à ceux de l'analyse d'échantillons témoins constitués de prélèvements soit sanguins soit cutanés préputiaux d'adultes sans antécédents. Il ressort de ce travail qu'il existe un polymorphisme du gène HOXA10 chez les adultes et chez les enfants, et que des altérations du gène HOXA10 sont présentes chez certains enfants ayant une TND, uni ou bilatéral. Cette identification de mutations du gène HOXA10 dans la population à TND traduit chez l'homme l'implication de ce gène dans le développement de l'appareil uro-génital. Toutefois, la place réelle de telles mutations dans la causalité de la non-descente du testicule nécessite d'autres études. 
L'ensemble de ces résultats est toutefois à rapprocher de travaux plus anciens [10] portant sur 35 fœtus et 58 enfants ayant un TND, et sur une population témoin de 22 foetus et 25 enfants normaux, tous âgés de 28 semaines à 3 ans. En l'absence de hernie inguinale, on retrouvait des malformations des reins, des uretères ou des vertèbres T10 à S5 chez $34 \%$ des fœtus et $18 \%$ des enfants ayant un TND. Ces résultats amenaient les auteurs à suggérer que la non-descente testiculaire pouvait résulter d'un développement anormal du champ développemental caudal de l'embryon [10].

\section{b) Le chromosome $Y$.}

Dans une étude récente [20], il n'a été retrouvé aucune microdélétion du bras long du chromosome $\mathrm{Y}$ (i.e. incluant le gène $\mathrm{DAZ}$ et la région AZF) dans une population de 42 adultes ayant eu dans l'enfance une orchidopexie pour nondescente testiculaire uni ou bilatérale. Ces patients présentaient pour $15 \%$ une azoospermie et pour $26 \%$ une oligospermie (numération $<20$ millions de spermatozoïdes $/ \mathrm{ml}$ ). De même, aucun de ces patients ne présentait de mutation du gène SRY, gène impliqué dans la différentiation sexuelle et le développement testiculaire. Les auteurs concluaient donc logiquement que la non-descente testiculaire n'est pas associée à des microdélétions des gènes DAZ, AZF ou SRY [20].

Cependant, à la même période paraissait dans une autre revue une étude similaire, à savoir la recherche de microdélétions du bras long du chromosome $\mathrm{Y}$ chez des patients ayant, entre autres, soit un antécédent de TND unilatéral, soit une infécondité idiopathique, tous étant soumis à un examen de sperme et une analyse histologique du tissu testiculaire prélevé bilatéralement par ponction à l'aiguille [22]. Les résultats de cette dernière étude [22] semblent en contradiction totale avec ceux de la précédente [20], puisqu'il en ressort qu'en cas de TND unilatéralement, une microdélétion du chromosome Y est retrouvée chez $28 \%$ (11/40) des patients ayant une oligospermie sévère (numération $<5$ millions de spermatozoïdes $/ \mathrm{ml}$ ) et chez aucun (0/20) des patients ayant une oligospermie modérée (numération= 10-20 millions de spermatozoïdes $/ \mathrm{ml}$ ).

Ces résultats sont cependant plus faciles à interpréter lorsqu'on comprend que les patients à testicule unilatéralement non descendu, avec moins de 5 millions de spermatozoïdes $/ \mathrm{ml}$, présentent une atrophie testiculaire bilatérale et une histologie à type d'absence de cellules germinales ou d'hypospermatogenèse sévère dans le testicule normalement descen$\mathrm{du}$, alors que ce dernier a une spermatogenèse normale dans de groupe de patient avec 10-20 millions de spermatozoïdes $/ \mathrm{ml}$. Les auteurs concluent de cette différence histologique (à l'âge adulte) que les testicules non descendus avec $10-20$ millions de spermatozoïdes/ml doivent en fait avoir eu une non-descente due à un obstacle mécanique, mais sans fournir aucune preuve de cette affirmation [22]. Il nous semblerait plutôt que le groupe avec moins de 5 millions de spermatozoïdes/ml est porteur d'au moins une autre pathologie en raison de l'atrophie testiculaire bilatérale ; un argument en faveur de notre hypothèse est que ces auteurs [22] retrouvent un taux semblable de microdélétions ( $26 \%$ ) chez 110 hommes dont l'infécondité idiopathique est associée à une azoospermie ou oligospermie sévère. Contrairement à ce qui semble paraître à première vue, les résultats de cette dernière étude [22] sont plutôt en accord avec ceux de la première étude [20], à savoir que la non-descente testiculaire ne semble pas être associée à des microdélétions du bras long du chromosome Y.

\section{c) Le chromosome 10.}

Des délétions du bras long du chromosome 10 ont été retrouvées chez des garçons présentant un TND unilatéral [52] ou bilatéral [62] associé à d'autres malformations lourdes. Des 10 cas rapportés dans la littérature, tous présentaient des anomalies génitales, incluant 4 cas avec un phénotype d'ambiguïté sexuelle et 9 cas de TND. Les auteurs [52] concluent que les délétions de l'extrémité du bras long du chromosome 10 sont toujours associées à une différentiation sexuelle anormale chez le mâle qui peut aller de la complète inversion sexuelle au micropénis et à la non-descente testiculaire. Il existe donc sur le bras long du chromosome 10 des gènes impliqués dans la différentiation sexuelle et la migration du testicule.

\section{d) Gène du récepteur aux androgènes.}

On sait à partir de travaux réalisés chez l'hu- 
main mâle âgé de 4 mois à 65 ans que le muscle crémaster possède des récepteurs aux androgènes, alors que le processus vaginal, le gubernaculum testis et le fascia sous-cutané en sont dépourvus [35]. On ne sait pas cependant si ces quatre structures possèdent ou non des récepteurs aux androgènes pendant la vie embryonnaire. Quoi qu'il en soit, une étude récente [71] montre à partir d'échantillons du sac herniaire (orchidopexie) ou du tissu préputial (circoncision) que le TND isolé n'est pas associé à des altérations du gène du récepteur aux androgènes. Ces résultats cependant n'apportent aucune information sur la vie fotale, car, comme l'indique les auteurs [71], le moment et le degré de l'expression des récepteurs aux androgènes dans les tissus cibles sont certainement très importants dans les processus de développement et de descente des testicules, comme cela a bien été montré chez les rongeurs.

\section{e) Gène INSL3.}

Insl3, aussi appelé facteur Leydig Insuline-like (Ley I-L) ou Relaxine-like (RLF), est un nouveau membre récemment caractérisé de la superfamille de l'hormone insuline. Insl3 est spécifiquement exprimé dans les cellules de Leydig du testicule fotal et adulte et dans les cellules thécales de l'ovaire post-natal $[72,53]$. Les analyses des transcipts d'Insl3 dans le testicule et l'ovaire au long de la vie pré- et postnatale de la souris, révèlent un profil sexuel dimorphique de l'expression d'Inls3 pendant le développement : aucun transcript d'Inls3 n'est détecté chez les embryons femelles quel que soit le stade, alors qu'ils sont détectés à J13 p.c. chez les embryons mâles $[72,53]$. Après la naissance, le niveau des transcipts d'Insl3 reste constant dans le testicule pendant les trois premières semaines, augmente au moment où la première vague de spermatides rondes entre en spermiogenèse, et atteint sa valeur la plus élevée dans le testicule adulte $[72,53]$.

Ces résultats ont soulevé l'hypothèse d'un rôle essentiel d'Insl3 dans la différentiation et la maintenance du phénotype mâle et de la spermatogenèse. La création de souris mutantes pour le gène Insl3 a montré que les mâles homozygotes mutants présentaient une non- descente testiculaire, avec localisation abdominale de ces derniers, les organes génitaux internes et externes étant par ailleurs normaux chez ces mâles normalement virilisés avec un comportement sexuel normal $[72,53]$. L'analyse histologique a permis de montrer que le développement du gubernaculum testis était fortement perturbé pendant le développement embryonnaire, et que cette perturbation était responsable de la non-descente testiculaire $[72,53]$. Les transcripts d'Insl3 sont exprimés dans le testicule en développement, mais pas dans le bulbe du gubernaculum ni dans aucun autre tissu de voisinage testiculaire chez la souris, ce qui est consistant avec l'idée que le testicule sécrète l'Insl3 et que le bulbe du gubernaculum est un organe cible [53]. Il ressort de ces résultats que Insl3 jouerait un rôle essentiel dans la descente testiculaire, et qu'il pourrait être le facteur encore non identifié spécifiquement impliqué dans le développement du gubernaculum testis.

La recherche des mutations du gène INSL3 chez 31 hommes ayant un antécédent uni ou bilatéral de TND, et chez 9 hommes témoins féconds sans antécédent de TND, n'a mis en évidence qu'une anomalie, à type de substitution, présente à une fréquence élevée dans les deux populations étudiées [40]. Ces données indiqueraient que le polymorphisme n'est pas relié au phénotype, et que les mutations du gène INSL3 ont peu de chance d'être une cause fréquente de la non-descente testiculaire chez l'homme [40]. Cependant, comme le font remarquer les auteurs [40], le phénotype des souris mutantes est une non-descente testiculaire avec localisation abdominale des testicules ; l'étude d'une population humaine avec un phénotypique semblable permettra de confirmer ou non l'implication du gène INSL3 dans la localisation abdominale persistante des testicules chez l'homme.

Il faut citer, par ailleurs, les travaux récents qui ont montré chez la souris que le diethylstilbestrol (DES), œstrogène synthétique responsable de non-descente testiculaire chez l'animal et l'homme, pourrait agir en altérant l'expression des ARNm d'Insl3 et par conséquent en perturbant le développement embryonnaire du gubernaculum testis [19]. 


\section{FACTeUrs de RisQue.}

\section{a) Environnementaux.}

Rappelons pour mémoire l'étude espagnole [23] qui établissait une association entre taux d'orchidopexie et niveaux de pesticides utilisés. Plus récemment [69], un risque de nondescente testiculaire a été rapporté chez les fils des mères travaillant dans le jardinage ou l'agriculture. En Hongrie, l'une des trois anomalies congénitales retrouvées avec une fréquence anormalement élevée chez les enfants de mères résidant dans un rayon de $25 \mathrm{~km}$ autour d'une usine chimique produisant de l'acrylonitrile, est le TND, avec un $\mathrm{OR}$ de 4,2 $(\mathrm{IC}=1,3-13,5)$ à $8,6(\mathrm{IC}=1,4-54,3)$; il existait d'autre part une diminution du risque de TND avec l'accroissement de la distance de la résidence à l'usine [15].

À noter aussi que l'exposition à la dioxine (2,3,7,8-tetrachlorodibenzo-p-dioxine) de fœtus porcins in vivo (une injection intra-fœetale directe) après le début de la différentiation génitale mais avant la croissance du gubernaculum testis, est associée à la naissance à une multiplication par trois (53\% versus $18 \%$ ) de la fréquence des anomalies génitales par rapport aux animaux témoins [3]. Si le taux de TND n'est pas différent entre exposés (4/15) et non exposés (3/17), les TND étaient abdominaux bilatéraux pour 3 exposés sur 4 mais en aucun cas chez les non exposés. Ces anomalies pourraient être dues à une régulation anormale de l'expression du récepteur aux œstrogènes par la dioxine [3].

En conclusion, des données s'accumulent peu à peu chez l'homme et l'animal sur un éventuel effet sur la migration testiculaire de substances chimiques de type disrupteurs endocriniens.

\section{b) Autres.}

Dans deux études épidémiologiques récentes $[70,2]$ portant sur les facteurs de risque de la "cryptorchidie" et de l'hypospadias, le poids à la naissance est le principal déterminant, comme déjà rapporté auparavant $[50,36]$. Le risque de " cryptorchidie" et d'hypospadias augmenterait avec une diminution du poids de naissance indépendamment [70] ou non [2] de l'âge gestationnel. Ces deux études concluent par ailleurs que la " cryptorchidie" et l'hypospadias partageraient en partie les mêmes étiologies.

\section{TRAITEMENTS DE LA NON DESCENTE TESTICULAIRE}

\section{Traitement chirurgical.}

"L'âge idéal pour l'orchidopexie " a été abaissé, par l'Académie Américaine de Pédiatrie, de la période de "après 4 ans et avant 6 ans " en 1975 à l'âge de “ 1 an ou peu après " en 1996 [1]. Outre les progrès réalisés dans les domaines de l'anesthésie et de la technique chirurgicale, cet abaissement de l'âge "idéal " pour l'orchidopexie repose essentiellement sur l'accumulation de données histologiques du testicule de l'enfant, qui ont mis en évidence les faits suivant : jusqu'à l'âge de un an, l'histologie du TND reste normale; le nombre de cellules germinales peut être réduit dans certains cas entre 1 et 2 ans ; au-delà de 2 ans, 30 à $40 \%$ des TND sont dépourvus de cellules germinales [45, 27, 49, 33, 48].

Si le nombre de cellules germinales est non différent dans le TND et dans le testicule controlatéral normalement descendu pendant la première année de vie, cela suggère que, quelle que soit par ailleurs la cause de la non-descente testiculaire, la position de non-descente pourrait représenter par elle même un facteur de réduction du nombre de cellules germinales après l'âge de 1 an (et donc une réduction potentielle de la fertilité future). La confirmation de cette hypothèse devrait être apportée dans plusieurs années lorsqu'un nombre suffisant d'enfants opérés avant l'âge de 2 ans atteindra un âge auquel le sperme et/ou la fertilité pourront être évalués.

À partir de l'analyse de 64 articles portant sur plus de 8000 testicules soumis à une orchidopexie, l'auteur [17] a évalué le taux de succès, défini comme étant " une position scrotale et une absence d'atrophie " lors d'un examen réalisé au moins 6 mois après l'intervention, à $74 \%$ pour les testicules abdominaux (qui représentent $34 \%$ du total), $87 \%$ pour les intra-canalaires et $92 \%$ pour ceux situés au- 
delà de l'anneau inguinal externe. La pauvreté des données n'a pas permis d'évaluer l'effet de l'âge du traitement sur le taux de succès. En conclusion de cette revue : échec de l'orchidopexie pour $25 \%$ des testicules abdominaux et pour $10 \%$ des testicules canalaires et au-delà, soit un échec global de l'orchidopexie dans environ $15 \%$ des cas. $5 \%$ des testicules descendus par orchidopexie [51] vont être ensuite retrouvés en situation non-descendue (échappement au traitement).

Une seule étude, à effectif réduit, fait état d'une absence de relation entre âge du traitement et volume testiculaire à l'âge adulte [65], alors qu'une étude précédente portant sur un effectif similaire faisait état d'une forte corrélation entre le volume testiculaire à l'âge adulte et sa position finale, i.e. à distance de l'orchidopexie [11]. Enfin, pas d'effet de l'âge du traitement sur le nombre de cellules germinales à partir de 2000 biopsies [25] confirmant des résultats précédents sur 670 biopsies [60].

\section{a) Testicule non descendu chez un indivi- du ayant fait sa puberté.}

En 1997, une équipe [61] rapportait la survenue d'une grossesse spontanée suivant une grossesse obtenue par FIV chez un couple dont le partenaire masculin avait subi à l'âge de 23 ans une orchidopexie pour un TND bilatéral en situation inguinale avec azoospermie et FSH sérique supérieure à $40 \mathrm{UI} /$. En s'appuyant sur un cas similaire rapporté quelques années auparavant [29], et sur le cas unique qu'ils rapportaient [61] les auteurs concluaient leur article en " recommandant de réaliser une orchidopexie chez les hommes adultes avec des TND palpables consultant pour infécondité, sans tenir compte des résultats des analyses de sperme ou des taux de FSH ". Bien que cet article ait été publié dans une respectable revue, on peut penser que le " reviewer" ait été quelque peu somnolent devant une conclusion aussi largement documentée!

En effet, il est classique que les patients pubères âgés de moins de 32 ans [21] se présentant avec un TND non traité aient plutôt une orchidectomie qu'une orchidopexie. La raison de cette conduite à tenir repose sur une étude de 1975 [46] selon laquelle le risque de décès pour cancer du testicule était supérieur à celui de décéder pendant l'orchidectomie. Cependant, la réduction, par les traitements cytotoxiques, de la mortalité liée au cancer du testicule a amené certaines équipes à reconsidérer cette question. C'est ainsi que dans 52 cas de TND chez des patients âgés de 15 à 66 ans, l'analyse histologique rétrospective de $l a$ totalité (et non pas d'un échantillon) de chacun des testicules enlevés a montré une absence complète de cellules germinales dans $69 \%$ des cas, un arrêt de maturation dans $29 \%$, une hypospermatogenèse dans $2 \%$ et une spermatogenèse normale dans aucun des cas [58]. Par contre deux cas $(4 \%)$ de carcinome in situ ont été trouvés. Les auteurs concluent que comme la grande majorité des TND chez les patients pubères ne contribuent pas à la fertilité et présentent un potentiel malin significatif, l'orchidectomie reste le traitement de choix.

Mais une publication récente [24] vient de rapporter deux nouveaux cas (âgés de moins de 32 ans) de TND bilatéraux en situation inguinales palpables, avec azoospermie et taux de FSH sérique dans les limites de la normale, pour lesquels une orchidopexie bilatérale a été suivie d'une reprise de la spermatogenèse se traduisant par des numérations de 1,6 à 8 millions de spermatozoïdes/ml dans l'année suivante, ce qui permit à ces deux couples un accès à la FIV.

On ne peut qu'être surpris par la discordance frappante entre d'une part les résultats de l'étude rétrospective portant sur 52 testicules analysés dans leur totalité et montrant l'absence de cellules germinales dans $70 \%$ des cas (incluant la majorité des testicules inguinaux), ce qui confirme a posteriori l'orchidectomie systématique réalisée dans cette équipe [58], et d'autre part les $100 \%$ de succès rapportés dans trois cas isolés [61, 24] à partir desquels les auteurs recommandent d'adopter leur conduite à tenir. On aurait pu penser que les résultats d'une étude multicentrique, s'appuyant sur des critères de sélection clairement définis et un effectif suffisant, représentaient un préalable indispensable avant toute modification d'une conduite à tenir (orchidectomie systématique) jusqu'alors argumentée et consensuelle... 
b) Les effets secondaires du traitement chirurgical peuvent être définis dans le domaine de la fertilité comme une réduction du gain attendu, réduction due à l'intervention ; ils ne recouvrent pas l'ensemble des complications chirurgicales survenant dans 5 à $12 \%$ des cas [51, 41]. Ces effets secondaires sont représentés par l'atrophie testiculaire, consécutive à une altération vasculaire, chez 1 à $2 \%$ des enfants [51,9], taux qui peuvent être plus élevés en cas de pédicule vasculaire insuffisamment long.

D'autres effets secondaires ont été plus récemment rapportés : on savait déjà par une étude expérimentale chez l'animal qu'une suture passée à travers l'albuginée entraînait une altération du parenchyme testiculaire [4]. Le fait de " placer une suture à travers le tissu testiculaire pour l'amener dans le scrotum et le fixer dans cette position ", est rapporté comme un facteur de risque d'infertilité dans une étude épidémiologique, indépendamment de la position pré opératoire du testicule [13]. Les résultats de cette étude sont renforcés par ceux d'une étude plus récente [67] montrant qu'une suture à travers l'albuginée lors de l'orchidopexie pré-pubertaire se traduisait dans $50 \%$ des cas à l'âge adulte par l'existence à l'échographie de micro-calcifications ou de microkystes sous l'albuginée, le parenchyme testiculaire étant par ailleurs normal.

L'évaluation échographique des testicules réalisée 2 à 11 ans après l'orchidopexie chez 68 enfants a montré que $47 \%$ des 75 testicules opérés étaient normaux en ce qui concerne la position, le volume, la structure du parenchyme et la perfusion artérielle; les $53 \%$ restants avaient l'un ou plusieurs des paramètres altérés, indépendamment de l'âge du traitement ou du temps écoulé depuis la chirurgie [56]. Cependant rien ne permet d'exclure pour le moment le fait que certaines de ces altérations pré-existaient au traitement.

Enfin, le traitement par orchidopexie seule ou précédée d'un échec du traitement hormonal réalisé à l'âge de 10 mois à 13 ans pour un TND uni ou bilatéral, est sans conséquence majeure sur le développement sexuel en ce qui concerne l'âge de la spermarche, de la première masturbation et du premier rapport sexuel par comparaison avec une population témoin [64]. Cependant, cette vision optimiste [64] n'exclut pas quelques difficultés certaines d'identification [47].

\section{EFFET DE L'ÂGE DU TRAITEMENT CHIRURGI- CAL SUR LES PARAMÈTRES DU SPERME.}

En cas de TND unilatéral :

- L'orchidopexie entre 2 et 12 ans a un effet positif sur la concentration et la mobilité des spermatozoïdes par rapport à l'absence de tout traitement [54] ;

- Aucun effet de l'âge du traitement sur la numération de spermatozoïdes n'a été montré pour des traitements chirurgicaux réalisés entre 1 mois et 19 ans $[54,63,42,14,26]$; une seule étude [44] rapporte une fréquence significativement plus grande de numération $>20$ millions $/ \mathrm{ml}$ quand l'orchidopexie est réalisée à 2-6 ans plutôt qu'à 6-10 ans ou à plus de 10 ans, mais il n'apparaît aucune différence significative entre les numérations moyennes de ces trois classes d'âge.

En cas de TND bilatéral :

- Une seule étude rapporte que l'orchidopexie avant 4 ans est associée à une meilleure "qualité " du sperme [63].

En conclusion, d'autres données, en particulier ceux de séries homogènes (diagnostic, technique chirurgicale...) et à effectifs suffisants, sont encore nécessaires pour confirmer ou infirmer un effet bénéfique du traitement précoce (2 ans) sur la production de spermatozoïdes et/ou la fertilité à l'âge adulte.

\section{Traitement hormonaL.}

Le traitement d'un TND par l'apport d'hormones exogènes de type hCG puis LHRH et analogues repose sur la notion que la cause la plus fréquente de la non-descente testiculaire est un défaut de la sécrétion prénatale d'androgènes consécutif soit à une sécrétion insuffisante de gonadotrophines hypophysaires, soit à une faible production de gonadotrophine par le placenta [32].

L'évaluation du taux de succès, défini comme un testicule dans le fond du scrotum à la fin du traitement, est rapportée dans une revue [55] 
de 33 articles publiés en anglais entre 1958 et 1990 , et montre que

1) ce taux est surévalué dans les études non randomisées,

2) dans les études randomisées $(n=11)$, les taux de succès sont : en cas d'exclusion des testicules rétractiles, de $12 \%$ avec LHRH (IC $=8$ - $15 \%$; 5 études, 335 testicules), de $19 \%$ avec hCG (IC $=13-25 \% ; 2$ études, 148 testicules) et de $5 \%$ avec placebo ( $\mathrm{IC}=2-7 \% ; 4$ études, 308 testicules); en cas d'inclusion des testicules rétractiles de $21 \%(\mathrm{IC}=18-24 \%)$ avec LHRH, $19 \%(\mathrm{IC}=13-25 \%)$ avec hCG et $4 \%$ (IC $=2-6 \%$ ) avec placebo.

Le taux de succès (incluant les études non randomisées) semble d'autant plus élevé que la position initiale du testicule est plus basse, ce qui est confirmé par des études plus récentes $[8,37,41]$. Dans les études randomisées $(n=$ 11), le taux de succès n'est pas différent quand le traitement est fait avant ou après l'âge de 4 ans [55]. Enfin, $24 \%$ (IC = $13-35 \%$ ) des testicules descendus à la fin du traitement ont été retrouvés en position non descendue (échappement au traitement) dans le suivi réalisé dans 5 des 11 études randomisées avec LHRH [55].

En résumé, un TND sur cinq descendra avec le traitement hormonal, mais un testicule sur quatre descendus par traitement hormonal reviendra en situation non-descendue par la suite.

\section{LES EFFETS SECONDAIRES DU TRAITEMENT} HORMONAL sont évoqués depuis longtemps puisque déjà en 1952 des altérations des tubules séminifêres étaient attribuées au traitement par hCG chez l'adulte [43]. Plus récemment, des travaux chez le rat adulte ont montré que l'injection d'hCG induisait une série de manifestations (infiltration des espaces interstitiels par les polynucléaires, augmentation de la perméabilité veineuse intra-testiculaire, augmentation du volume de liquide interstitiel...) analogues à une réponse inflammatoire aiguë $[6,68,30]$. Ces altérations de l'espace interstitiel seraient responsables des atteintes focalisées de la spermatogenèse, à type d'arrêt complet, observés sur les biopsies testiculaires réalisées 12 heures [38] à 10 jours [66] suivant une injection d'hCG.
Chez l'enfant, des études récentes attirent l'attention sur des effets secondaires possibles du traitement par hCG ou $\mathrm{GnRH}$ et analogues. Ainsi, trois types d'effets sont rapportés sur l'analyse des biopsies testiculaires : accroissement du saignement dans l'espace interstitiel $[31,37,16]$, augmentation de l'apoptose des cellules germinales $[28,18]$, diminution du nombre moyen de spermatogonies par tubules séminifêres chez les enfants âgés de 1 à 3 ans $[41,12]$. Les résultats de ces études sont toutefois à prendre avec précaution pour deux raisons au moins : 1) toutes ces données proviennent de biopsies testiculaires réalisées chez des enfants dont le traitement hormonal a été un échec, ce qui peut constituer un biais de sélection dans la mesure où on ignore si de telles altérations existent aussi en cas de réussite du traitement ; 2) l'âge auquel est réalisé le traitement hormonal est aussi très important en raison de la maturation neuroendocrine du testicule de l'enfant ; ainsi, un traitement par hCG sans succès sur la descente testiculaire ne modifie pas le nombre de cellules germinales par tubule séminifère quand le traitement est réalisé entre 4 et 5 ans [7] ou entre 10 et 11 ans [11]. La seule conclusion qui puisse être tirée de ces études, à l'heure actuelle, est de ne pas faire de traitement hormonal entre 1 et 3 ans chez les enfants ayant un TND [12].

\section{RÉFÉRENCES}

1. AMERICAN ACADEMY OF PEDIATRICS : Timing of elective surgery on the genitalia of male children with particular reference to the risks, benefits, and psychological effects of surgery and anesthesia. Pediatrics, 1996, $97: 590-594$.

2. AKRE O., LIPWORTH L., CNATTINGIUS S., SPAREN P., EKBOM A. : Risk factor patterns for cryptorchidsm and hypospadias. Epidemiology, 1999, 10 : 364-369.

3. BARTHOLD J.S., KRYGER J.V., DERUSHA A.M., DUEL B.P., JEDNAK R., SKAFAR D.F. : Effects of an environmental endocrine disruptor on fetal development, estrogen receptor alpha and epidermal growth factor receptor expression in the porcine male genital tract. J. Urol., 1999, $162: 864-871$.

4. BELLINGER M.F., ABROMOWITZ H., BRANTLEY S. et al. : Orchidopexy : An experimental study of the effect of surgical technique on testicular histology. J. Urol., 1989, $142: 553-555$. 
5. BENSON G.V., LIM H., PARIA B.C., SATOKATA I., DEY S.K., MAAS R.L. : Mechanisms of reduced fertility in Hoxa-10 mutant mice : uterine homeosis and loss of maternal Hoxa-10 expression. Dev., 1996, 122 : 2687-2696.

6. BERGH A., ROOTH P., WIDMARK A., DAMBER J.E. : Treatment of rats with hCG induces inflammation-like changes in testicular microcirculation. J. Reprod. Fertil., 1987, 79 : 135-143.

7. BICA D.T.G., HADZISELIMOVIC F. : Buserelin treatment of cryptorchidism : a randomized, doubleblind, placebo-controlled stuḍy. J. Urol., 1992, 148 : 617-620.

8. CHRISTIANSEN P., MULLER J., BUHL S. et al. : Hormonal treatment of cryptorchidism-hCG or GnRH-a multicentre study. Acta Pediatr., 1992, 81 : 605-608.

9. CORTES D. : Cryptorchidism : Aspects of pathogenesis, histology and treatment. $\mathrm{PhD}$ Thesis, Copenhagen, 1998, 1-54.

10. CORTES D., THORUP J.M., BECK B.L. : Quantitative histology of germ cells in the undescended testes of human fetuses, neonates and infants. J. Urol., 1995, 154 : 1188-1192.

11. CORTES D., THORUP J.M., LINDENBERG S. : Fertility potential after unilateral orchiopexy : simultaneous testicular biopsy and orchiopexy in a cohort of 87 patients. J. Urol., 1996, 155 : 1061-1063.

12. CORTES D., THORUP J.M., VISFELDT J. : Hormonal treatment may harm the germ cells in 1 to 3-year-old boys with cryptorchidism. J. Urol., 2000, 163 : 1290-1292.

13. COUGHLIN M.T., BELLINGER M.F., LAPORTE R.E., LEE P.A. : Testicular suture : a significant risk factor for infertility among formerly cryptorchid men. J. Pediatr. Surg., 1998, 33 : 1790-1793.

14. COUGHLIN M.T., BELLINGER M.F., LEE P.A. : Age at unilateral orchiopexy : effect on hormone levels and sperm count in adulthood. J. Urol., 1999, 162 : 986-989.

15. CZEIZEL A.E., HEGEDÜS S., TIMAR L. : Congenital abnormalities and indicators of germinal mutations in the vicinity of an acrylonitrile producing factory. Mut. Res., 1999, 427 : 105-123.

16. DEMIRBILEK S., ATAYURT H.F., CELIK N., AYDIN G. : Does treatment with human chorionic gonadotropin induce reversible changes in undescended testes in boys? Pediatr. Surg. Int., 1997, 12 : 591-594.

17. DOCIMO S.G. : The results of surgical therapy for cryptorchidism : a literature review and analysis. J. Urol., 1995, 154 : 1148-1152.

18. DUNKEL L., TASKINEN S., HOVATA O., TILLY J.L., WIKSTRÖM S. : Germ cell apoptosis after treatment of cryptorchidism with human chorionic gonadotropin is associated with impaired reproductive function in the adult. J. Clin. Invest., 1997, 100 : 2341-2346.
19. EMMEN J.M.A., MCLUSKEY A., ADHAM I.M. et al. : Involvement of Insulin-Like Factor 3 (Insl3) in Diethylstilbestrol-induced cryptorchidism. Endocrinology, 2000, 141 : 846-849.

20. FAGERLI J., SCHNECK F.X., LEE P.A., BELLINGER M.F., WITCHEL S. : Absence of microdeletions in the $\mathrm{Y}$ chromosome in patients with a history of cryptorchidism and azoospermia or oligospermia. Fertil. Steril., 1999, $71: 697-700$.

21. FARRER J.H., WALKER A.H., RAJFER J. : Management of the postpubertal cryptorchid testis: a statistical review. J. Urol., 1985, 134 : 1071-1076.

22. FORESTA C., MORO E., GAROLLA A ., ONISTO M., FERLIN A. : Y chromosome microdeletion in cryptorchidism and idiopathic infertility. J. Clin. Endocrinol. Metab., 1999, 84 : 3660-3665.

23. GARCIA-RODRIGUEZ J., GARCIA-MARTIN M., NOGUERAS-OANA M. et al. : Exposure to pesticides and cryptorchidism : geographical evidence of a possible association. Environ. Health Perspect., 1996, 104 : 1090-1095.

24. GIWERCMAN A., HANSEN L.L., SKAKKEBAEK N.E. : Initiation of sperm production after bilateral orchiopexy : clinical and biological implications. J. Urol., 2000, 163 : 1255-1256.

25. GRACIA J., GONZALEZ N., GOMEZ M.E., PLAZA L., SANCHEZ J., ALBA J. : Clinical, and anatomical study of 2000 cryptorchid testes. J. Urol., 1995, 75 : 697-701.

26. GRACIA J., SANCHEZ ZALABARDO J., SANCHEZ GARCIA J., GARCIA C., FERRANDEZ A. : Clinical, physical, sperm and hormonal data in 251 adults operated on for cryptorchidism in childhood. B. J. U. Int., 2000, 85 : 1100-1103.

27. HADZISELIMOVIC F., HERZOG B., BUSER M. : Development of cryptorchid testes. Eur. J. Pediatr., 1987, 146 (Suppl 2) : S8-S12.

28. HEISKANEN P., BILLIG H., TOPPARI J. et al. : Apoptotic cell death in the normal and cryptorchid human testis : the effect of human chorionic gonadotropin on testicular cell survival. Pediatr. Res., 1996, 40 : 351-356.

29. HEATON N.D., DAVENPORT M., PRYOR J.P. : Fertility after correction of bilateral undescended testes at the age of 23 years. Brit. J. Urol., 1993, 71 : 490-491.

30. HJERTKVIST M., BERGH A., DAMBER J.-E. : HCG treatment increases intratesticular pressure in the abdominal testis of unilaterally cryptorchid rats. J. Androl., 1988, 9 : 116-120.

31. HJERTKVIST M., LÄCKGREN G., PLOËN L., et al. : Does hCG treatment induce inflammation-like changes in undescended testes in boys? J. Pediatr. Surg., 1993, 28 : 254-257.

32. HOSIE S., WESSEL L., WAAG K.L. : Could testicular descent in humans be promoted by direct androgen 
stimulation of the gubernaculum testis ? Eur. J. Pediatr. Surg., 1999, $9: 37-41$.

33. HUFF D.S., HADZISELIMOVIC F., SNYDER H.M., DUCKETT J.W., KEATING M.A. : Postnatal testicular maldevelopment in unilateral cryptorchidism. J. Urol., 1989, 142 : 546-548.

34. HUTSON J.M., HASTHORPE S., HEYNS C. : Anatomical and functional aspects of testicular descent and cryptorchidism. Endocr. Rev., 1997, 18 : 259280.

35. JOHANSEN T.E.B., KLEIN H. : Evidence of androgen receptivity in the pathway of testicular descent in humans. Eur. Urol., 1993, 23 : 466-468.

36. JONES M.E., SWERDLOW A.J., GRIFFTH M., GOLDACRE M.J. : Prenatal risk factors for cryptorchidism : a record linkage study. Paed. Perin. Epid., 1998, $12: 383-396$.

37. KALEVA M., ARSALO A., LOUHIMO I., et al : Treatment with human chorionic gonadotrophin for cryptorchidism : clinical and histological effects. Int. J. Androl., 1996, 19 : 293-298.

38. KERR J.B., SHARPE R.M. : Focal disruption of spermatogenesis in the testis of adult rats after a single administration of human chorionic gonadotrophin. Cell Tissue Res., 1989, 257 : 163-169.

39. KOLON T.F., WIENER J.S., LEWITTON M., ROTH R.D., GONZALES E.D., LAMB D.J. : Analysis of homeobox gene HOXA10 mutations in cryptorchidism. J. Urol., 1999, $161: 275-280$.

40. KRAUSZ C., QUINTANA-MURCI L., FELLOUS M., SIFFROI J.P., MCELREAVEY K. : Absence of mutations involving the INSL3 gene in human idiopathic cryptorchidism. Mol. Hum. Reprod., 2000, 6 : 298302.

41. LALA R., MATARAZZO P., CHIABOTTO P. et al. : Early hormonal and surgical treatment of cryptorchidism. J. Urol., 1997, 157 : 1898-1901.

42. LENZI A., GANDINI L., LOMBARDO F. et al : Unilateral cryptorchidism corrected in prepubertal age : evaluation of sperm parameters, hormones, and sperm antibodies in adult age. Fertil. Steril., 1997, 67 : 943-948.

43. MADDOCK W.O., NELSON W.O. : The effects of chorionic gonadotrophin in adult men : increase estrogen and 17-ketosteroid excretion, gynecomastia, Leydig cell stimulation and seminiferous tubule damage. J. Clin. Endocrinol. Metab., 1952, 12 : 985-1007.

44. MANDAT K.M., WIECZORKIEWICZ B., GUBATAKACATA M., SYPNIEWSKI J., BUJOK G. : Semen analysis of patients who had orchidopexy in childhood. Eur. J. Pediatr. Surg., 1994, 4 : 94-97.

45. MANGEL W., HEINZ H.A., SIPPEL W.G., HECKER W.C. : Studies on cryptorchidism : a comparison of histological findings in the germinative epithelium before and after the second year of life. J. Pediatr. Surg., 1974, 9 : 445-450.
46. MARTIN D.C., MENCK H.R. : The undescended testis : management after puberty. J. Urol., 1975, 114 : 77-85.

47. MASI G., MILLEPIEDI S., BROVEDANI P., FAVILLA L., MUCCI M. : Psychopathological aspects of cryptorchidism in children and adolescents. Ch. Psych. Hum. Dev., 1999, 30 : 75-85.

48. MCALEER I.M., PACKER M.G., KAPLAN G.W., SCHERZ H.C., KROUS H.F., BILLMAN G.F. : Fertility index analysis in cryptorchidism. J. Urol., 1995, $153: 1255-1258$.

49. MININBERG D.T., RODGER J.C., BEDFORD J.M. : Ultrastructural evidence of the onset of testicular pathological conditions in the cryptorchid human testis within the first year of life. J. Urol., 1982, 128 : 782-788.

50. MÖLLER H., SKAKKEBAEK N.E. : Testicular cancer and cryptorchidism in relation to prenatal factors : case-control studies in Denmark. Cancer Causes and Control, 1997, $8:$ 904-912.

51. MOUL J.W., BELMAN A.B. : A review of surgical treatment of undescended testes with emphasis on anatomical position. J. Urol., 1988, 140 : 125-128.

52. MUTOH A., SASAGAWA I., TATENO T., SAWAMURA T., NAKADA T. : Long arm deletion of chromosome 10 in a boy with monorchidism. Scand. J. Urol. Nephrol., 1999, 33 : 77-78.

53. NEF S., PARADA L.F. : Cryptorchidism in mice mutant for Insl3. Nat. Genet,, 1999, 22 : 295-299.

54. OKUYAMA A., NONOMURA N., NAKAMURA M. et al. : Surgical management of undescended testis : retrospective study of potential fertility in 274 cases. J. Urol., 1989, 142 : 749-751.

55. PYÖRÄLÄ S., HUTTUNEN N.P., UHARI M. : A review and meta-analysis of hormonal treatment of cryptorchidism. J. Clin. Endocrinol. Metab., 1995, $80: 2795-2799$.

56. RIEBEL T., HERMANN C., WIT J., SELLIN S. : Ultrasonographic late results after surgically treated cryptorchidism. Pediatr. Radiol., 2000, 30 : 151-155.

57. RIJLI F.M., MATYAS R., PELLEGRINI M. et al. : Cryptorchidism and homeotic transformations of spinal nerves and vertebrae in Hoxa-10 mutant mice. Proc. Natl. Acad. Sci. USA, 1995, 92 : 8185-8189.

58. ROGERS E., TEHAN S., GALLAGER H. et al. : The role of orchiectomy in the management of postpubertal cryptorchidism. J. Urol., 1998, 159 : 851-854.

59. SATOKATA I., BENSON G., MAAS R. : Sexually dimorphic sterility phenotypes in Hoxa10-deficient mice. Nature, 1995, $374: 460-463$.

60. SCHINDLER A.M., DIAZ P., CUENDET A., SIZONENKO P.C. : Cryptorchidism : a morphological study of 670 biopsies. Helv. Paediat. Acta, 1987, 42 : 145-158.

61. SHIN D., LEMACK G.E., GOLDSTEIN M. : 
Induction of spermatogenesis and pegnancy after adult orchiopexy. J. Urol., 1997, $158: 2242$.

62. SUZUKI Y., SASAGAWA I., NAKADA T., ONMURA Y. : Bilateral cryptorchidism associated with terminal deletion of 10q. Urol. Int., 1998, 61 : 186-187.

63. TASKINEN S., HOVATA O., WIKSTRÖM S. : Early treatment of cryptorchidism, semen quality and testicular endocrinology. J. Urol, 1996, $156: 82-84$.

64. TASKINEN S., HOVATA O., WIKSTRÖM S. : Sexual development in patients treated for cryptorchidism. Scand. J. Urol. Nephrol., 1997, 31 : 361-364.

65. TASKINEN S., WIKSTRÖM S. : Effect of age at operation, location of testis and proliferative hormonal treatment on testicular growth after cryptorchidism. J. Urol., 1997, $158:$ 471-473.

66. VAN VLIET J., ROMMERTS F.F.G., DEROOIJ D.G., BUWALDA G., WENSING C.J.G. : Reduction of testicular blood flow and focal degeneration of tissue in the rat after administration of human chorionic gonadotrophin. J. Endocrinol., 1988, 117 : 51-57.

67. WARD J.F., CILENTO B.G., KAPLAN G.W., VELLING T., PUCKETT M., STOCK J. : The ultrasonic description of post pubertal testicles in men who have undergone prepubertal orchiopexy for cryptorchidism. J. Urol., 2000, $163: 1448-1450$.

68. WIDMARK A., BERGH A., DAMBER J.E., SMEDEGARD G. : Leucocytes mediate the hCG-induced increase in testicular venular permeability. Mol. Cell. Endocrinol., 1987, $53: 25-31$.

69. WEIDNER I.S., MÖLLER H., JENSEN K.T., SKAKKEBAEK N.E. : Cryptorchidism and hypospadias in sons of gardners and farmers. Environ. Health Perspect., 1998, 106 : 793-796.

70. WEIDNER I.S., MÖLLER H., JENSEN K.T., SKAKKEBAEK N.E. : Risk factors for cryptorchidism and hypospadias. J. Urol., 1999, 161 : 1606-1609.

71. WIENER J.S., MARCELLI M., GONZALES E.T., ROTH D.R., LAMB D.J. : Androgen receptor gene alterations are not associated with isolated cryptorchidism. J. Urol., 1998, $160:$ 863-865.

72. ZIMMERMANN S., STEDING G., EMMEN J.M.A. et $a l$. : Targeted disruption of the Insl3 gene causes bilateral cryptorchidism. Mol. Endocrinol., 1999, 13 : 681-691.

\title{
Maldescended Testis: causes and treatments
}

\author{
R. MIEUSSET
}

